\title{
Extended Bernoulli equation, friction loss, and friction coefficient for microscopic Jeffery-Hamel flow with small Reynolds number up to $\mathbf{O}(1)$
}

\author{
Toshihide FUJIKAWA*, Ryu EGASHIRA**, Shigeo FUJIKAWA***, Kazu TAKEDA**** \\ and Tetsuya KODAMA**** \\ *Department of Mechanical Engineering, Miyakonojo College, National Institute of Technology \\ 473-1 Yoshio-cho, Miyakonojo, Miyazaki 885-8567, Japan \\ E-mail: fujikawa@cc.miyakonojo-nct.ac.jp \\ **Department of Intelligent Mechanical Engineering, Fukuoka Institute of Technology \\ 3-30-1 Wajiro-higashi, Higashi-ku, Fukuoka 811-0295, Japan \\ ${ }^{* \star *}$ Hokkaido University \\ Kita 8, Nishi 5, Kita-ku, Sapporo, Hokkaido 060-0808, Japan \\ ${ }^{* * * \star}$ Department of Biomedical Engineering, Graduate School of Biomedical Engineering, Tohoku University \\ 4-1 Seiryou-cho, Aoba-ku, Sendai 980-8575, Japan
}

Received 12 July 2016

\begin{abstract}
The extended Bernoulli equation is formulated in an exact form for a microscopic and small Reynolds number Jeffery-Hamel flow in a two-dimensional convergent or divergent channel. The friction loss and the friction coefficient derived from the extended Bernoulli equation are also obtained for the purpose of engineering applications. The assumption of microscopic and low Reynolds number flow enables us to make the analysis simple, and the results obtained are expressed in forms easy to use. The zeroth- and first-order approximate solutions of velocity distribution in the channel are obtained by solving the nonlinear ordinary differential equation with the optimal homotopy asymptotic method. The zeroth-order solution is shown to be the same function form as that in the two-dimensional parallel flow, i.e., the two-dimensional Poiseuille flow. The extended Bernoulli equation, the friction loss, and the friction coefficient in a finite region of the channel, which are indispensable for applications, are reasonably derived along a stream line and also expressed by cross-sectional average quantities. The cross-sectional average formulae of the friction loss and the friction coefficient are expressed by the geometry of the channel, i.e., the convergent or divergent angle, the channel length, channel widths at inlet and exit. These formulae include the corresponding well-known ones for the two-dimensional parallel flow as a special case where the angle is zero. The friction coefficient drastically increases according to the increase in the angle, especially in a narrow channel region, and attains more than ten times of the friction coefficient for the parallel flow.
\end{abstract}

Key words : Jeffery-Hamel flow, Two-dimensional parallel flow, Microscopic viscous flow, Low Reynolds number flow, Extended Bernoulli equation, Friction loss, Friction coefficient

\section{Introduction}

Flows of incompressible viscous fluid in convergent or divergent channels are fundamental and applicable problems in many fields, such as pharmaceutical manufacturing and biomechanical, chemical, mechanical, and civil engineering. One of such flows is the well-known Jeffery-Hamel flow in the case where the two-dimensional channel is composed of two semi-infinite convergent or divergent planes, the intersecting line of which is a sink or a source. This flow was first studied by Jeffery in 1915 and by Hamel in 1916, and since then the Jeffery-Hamel flow has attracted a large number of researchers because it has provided both interesting mathematical and physical problems, such as the construction of analytical solutions of the nonlinear ordinary differential equation governing the flow (Millsaps and 
Pohlhausen, 1953; Stow et al., 2001; Esmaeilpour and Ganji, 2010) and bifurcation structures (Banks et al., 1988; Goldshtik et al., 1991; Haines et al., 2011; Khan et al., 2013): these studies have mainly focused on large Reynolds number flows in the divergent channels because complexities of such flows have attracted researchers.

Paying attention to a microscopic Jeffery-Hamel flow with small Reynolds number, on the other hand, we notice that the flow has not yet been fully unraveled even at the present time especially from the view point of applications, although it is much simpler than the larger Reynolds number flows. For example, we have not any formulae, such as extended Bernoulli equation, friction loss, i.e., pressure loss due to viscosity, and friction coefficient; these terminology follows the textbook by Sabersky, Acosta, and Hauptmann (1971). Due to this, it has not been possible for us to estimate the pressure distribution along the flow in convergent or divergent channels even for a very small Reynolds number. In fact, the authors have encountered difficulty in evaluating in a theoretical way the friction loss in microscopic lymphatic vessels of mice being used for the investigation of lymphatic drug delivery system (Okuno et al., 2013; Shao et al., 2013; Sato et al., 2014; Kato et al., 2015; Kodama et al., 2015; Kodama et al., 2016). The lymphatic vessel flow is a flow in a microscopic tapered tube and therefore not two-dimensional. However, even the two-dimensional flow like the Jeffery-Hamel flow could be quite helpful in interpreting the authors' experiment. This is the reason why the authors have begun buckling down to the study on the friction loss and the friction coefficient of the Jeffery-Hamel flow. For reference, typical dimensions of size and velocity in the lymphatic vessels are respectively around $200 \mu \mathrm{m}$ in diameter and less than $1 \mathrm{~mm} / \mathrm{s}$; therefore, the flow is very microscopic and Reynolds number is much smaller than or at the largest around unity.

The aim of this paper is to formulate the extended Bernoulli equation containing the friction loss in an exact form and the friction coefficient of the microscopic Jeffery-Hamel flow with small Reynolds number of the order of unity or less than it. In the followings, in Subsection 2.2, we first find a fundamental relation among Reynolds number $R e$, dimensionless flow velocity $u_{0}$ (=constant) along the symmetrical axis of the channel, and convergent or divergent angle $\alpha$ and obtain the formula $R e=4 \alpha u_{0}$. This is different from the relation, $R e=6 \alpha u_{0}$, which is known for large Reynolds number flow (Landau and Lifshitz, 1987). Furthermore, we derive the extended Bernoulli equation. In Subsection 2.3, velocity distribution in the channel is obtained by solving the nonlinear ordinary differential equation. In Subsection 2.4, the extended Bernoulli equation is applied to describe the friction loss in a finite region of the channel, by which the friction coefficient is appropriately defined in the form analogous to that of the two-dimensional parallel flow and the coefficient of the Jeffery-Hamel flow is found to reduce to that of the parallel flow as the limiting case of $\alpha \rightarrow 0$. In Section 3, the friction loss and the friction coefficient are quantitatively evaluated, and it is shown that these are greatly influenced by the convergent or divergent angle, especially in a narrow channel region. Section 4 is the summary.

\section{Flow between two intersecting planes}

\subsection{Governing equations and boundary conditions}

This subsection summarizes governing equations and boundary conditions for the Jeffery-Hamel flow following Landau and Lifshitz's textbook (1987) for the purpose of making readers understand this paper's complicated contents easily. As shown in Fig. 1, let us consider a convergent steady incompressible viscous flow between two intersecting, semi-infinite planes inclined at an angle $\alpha$ to each other, with a plane of symmetry for the flow at $z=0$ (Jeffery, 1915; Hamel, 1916); all results below hold for a divergent flow if the sign of all velocities except $u_{0}(=$ a positive constant) is inverted. A cylindrical-polar coordinate system $(r, \phi, z)$ centered on the origin $O$ is adopted. The flow is directed toward the origin $O$ and entirely radial, i.e.,

$$
v_{r}=v(r, \phi), v_{\phi}=0, v_{z}=0
$$

Then, a set of governing equations is given by

$$
\begin{aligned}
& \frac{\partial(r v)}{\partial r}=0 \\
& -\frac{1}{\rho r} \frac{\partial p}{\partial \phi}+\frac{2 v}{r^{2}} \frac{\partial v}{\partial \phi}=0,
\end{aligned}
$$




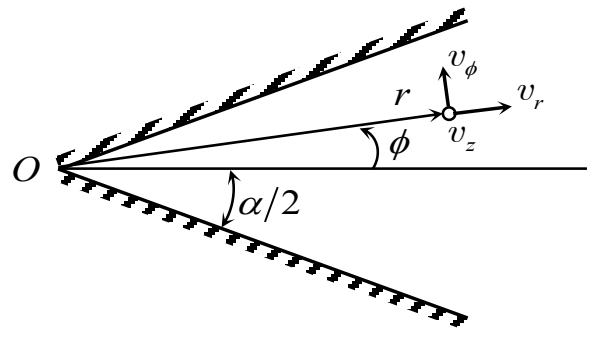

Fig. 1 Cylindrical-polar coordinates for the Jeffery-Hamel flow. The $z$ axis is the line of intersection of the planes and is denoted by $O$. The flow is radially directed toward the origin $O$.

$$
v \frac{\partial v}{\partial r}=-\frac{1}{\rho} \frac{\partial p}{\partial r}+v\left(\frac{\partial^{2} v}{\partial r^{2}}+\frac{1}{r^{2}} \frac{\partial^{2} v}{\partial \phi^{2}}+\frac{1}{r} \frac{\partial v}{\partial r}-\frac{v}{r^{2}}\right)
$$

where $p$ is the pressure of fluid, $\rho$ the density, and $v$ the kinematic viscosity (=constant). From Eq. (2), $r v$ is found to be a function of $\phi$ alone. Introduction of the following dimensionless function

$$
u(\phi)=\frac{r v}{6 v}
$$

enables us to obtain from Eq. (3)

$$
\frac{1}{\rho} \frac{\partial p}{\partial \phi}=\frac{12 v^{2}}{r^{2}} \frac{\mathrm{d} u}{\mathrm{~d} \phi}
$$

The integration of Eq. (6) with respect to $\phi$ gives

$$
\frac{p}{\rho}=\frac{12 v^{2}}{r^{2}} u(\phi)+\Psi(r),
$$

where $\Psi(r)$ is an unknown function to be sought below. Substitution of Eq. (7) into Eq. (4) gives us

$$
\frac{\mathrm{d}^{2} u}{\mathrm{~d} \phi^{2}}+4 u+6 u^{2}=\frac{1}{6 v^{2}} r^{3} \Psi^{\prime}(r)
$$

in which $\Psi^{\prime}(r)=\mathrm{d} \Psi(r) / \mathrm{d} r$. The left-hand side of this equation is the function of only $\phi$, on the other hand, the right-hand side the function of $r$ alone, therefore each must be a constant value, which will be denoted by $2 A$. Thus, $\Psi(r)=-6 v^{2} A / r^{2}+$ constant, by which we have the following two equations

$$
\begin{aligned}
& u^{\prime \prime}+4 u+6 u^{2}=2 A, \\
& \frac{p}{\rho}=\frac{6 v^{2}}{r^{2}}(2 u-A)+\text { constant }
\end{aligned}
$$

where $u^{\prime}=\mathrm{d} u(\phi) / \mathrm{d} \phi$ and $u^{\prime \prime}=\mathrm{d}^{2} u(\phi) / \mathrm{d} \phi^{2}$. It should be noted in Eq. (10) that the pressure $p$ is the function of variables $r$ and $\phi$, which is different from the pressure in the two-dimensional parallel flow through a straight channel, i.e., the two-dimensional Poiseuille flow (Levich, 1962; Schlichting, 1968): the pressure gradient along the flow is constant in the parallel case. The function $u(\phi)$ obeys the following boundary condition and mass conservation equation:

$$
u\left( \pm \frac{1}{2} \alpha\right)=0
$$




$$
Q=6 v \rho \int_{-\alpha / 2}^{\alpha / 2} u \mathrm{~d} \phi
$$

where $Q$ is the mass flow rate at a cross section at an arbitrary distance $r$ from the origin $O$, and it takes a positive value for the case where the line of intersection of the planes is a source, while a negative value for a sink. For the source, we have flow in a divergent channel, and for the sink, we have flow in a convergent channel.

From here, our principal interest is in the convergent flow with a very small velocity which we have encountered in the study of a microscopic lymphatic flow of mice as mentioned in Introduction. In such a situation, from Eqs. (9) and (12), we have

$$
\begin{aligned}
& \alpha=\int_{-u_{0}}^{0} \frac{\mathrm{d} u}{\sqrt{\left(u+u_{0}\right)\left[-u^{2}-\left(1-u_{0}\right) u+B\right]}}, \\
& \operatorname{Re}=-6 \int_{-u_{0}}^{0} \frac{u \mathrm{~d} u}{\sqrt{\left(u+u_{0}\right)\left[-u^{2}-\left(1-u_{0}\right) u+B\right]}},
\end{aligned}
$$

where $R e$ is the Reynolds number defined by $|Q| /(\rho v)$, the constant $B$ is positive and $B=A+u_{0}$, the relation $u(\phi)=u(-\phi)$ is assumed, and the function $u(\phi)$ is negative everywhere and decreases monotonically from $u=0$ at $\phi= \pm \alpha / 2$ to $u=-u_{0}<0$ at $\phi=0 ; u_{0}$ is the maximum constant value of $|u|$. At $\phi=0, \mathrm{~d} u / \mathrm{d} \phi=0$ holds.

\subsection{Extended Bernoulli equation and friction loss}

Let us here start discussion from the possibility of integrating Eqs. (13) and (14) in any simple forms. A very rough typical magnitude of $u_{0}$ should be estimated for the flow condition such as $\alpha=32^{\circ}$ and $|Q|=3 \times 10^{-5} \mathrm{~kg} / \mathrm{s}$; the angle $\alpha$ is basically expressed by radian unit, but it is occasionally done by degree unit for helping intuitive understanding. In such cases, degree unit symbol $\left({ }^{\circ}\right)$ is attached. These values are determined by the visualization of an actual lymphatic vessel flow and the CAD (Computer-Aided Design) analysis of the visualized image. Then, we have $u_{0} \approx 0.05$. This value of $u_{0}$ is a rough one obtained by assuming that the flow is parallel through a straight channel. In a later stage, $u_{0}$ is shown to be constant along the symmetrical axis of the channel. This value of $u_{0}$ is found to be very small compared with unity, and it enables us to approximately carry out the integration of Eqs. (13) and (14); approximation, $-u^{2}-\left(1-u_{0}\right) u+B \approx B-u$, holds for a large positive value of $B$. This approximation will be justified below. Then, Eqs. (13) and (14) can be easily integrated to be

$$
\begin{aligned}
& \alpha=2 \sqrt{\frac{u_{0}}{B+u_{0}}}\left[1+\frac{1}{6}\left(\sqrt{\frac{u_{0}}{B+u_{0}}}\right)^{2}+\frac{3}{40}\left(\sqrt{\frac{u_{0}}{B+u_{0}}}\right)^{4}+\cdots\right], \\
& \operatorname{Re}=8 u_{0} \sqrt{\frac{u_{0}}{B+u_{0}}}\left[1+\frac{1}{10}\left(\sqrt{\frac{u_{0}}{B+u_{0}}}\right)^{2}+\frac{1}{24}\left(\sqrt{\frac{u_{0}}{B+u_{0}}}\right)^{4}+\cdots\right] .
\end{aligned}
$$

Evaluation of $B$ by the use of numerical values of $\alpha$ and $u_{0}$ given above gives $B \approx 11.8 u_{0}$ and $R e \approx 0.026$. Therefore, the value of $B / u_{0}$ is much larger than unity. To make sure, comparing approximate values of $\alpha$ and $R e$ in Eqs. (15) and (16) with numerically integrated values of Eqs. (13) and (14) for $u_{0}=0.5$ and $B / u_{0}=6$, which are limiting values for large $u_{0}$, we have respectively $\alpha=0.5757$ and $R e=1.15$ from Eqs. (13) and (14), while $\alpha=0.5548$ and $R e=1.11$ from the leading terms of Eqs. (15) and (16). Therefore, the leading terms of Eqs. (15) and (16) can provide us good approximations for $\alpha$ and $R e$, and these will be used below.

It is convenient for us to express $B$ by the function of $\alpha$ using the leading term of Eq. (15) as

$$
B=\frac{4-\alpha^{2}}{\alpha^{2}} u_{0} \quad(\alpha \neq 0)
$$

whence

$$
R e=4 \alpha u_{0} .
$$


Equation (18) is different from the one given in Landau and Lifshitz's textbook (1987), $R e=6 \alpha u_{0}$, for large $u_{0}$ where $|u|$ differs appreciably from $u_{0}$ only for $\phi$ close to $\pm \alpha / 2$, i.e., in the immediate neighborhood of the walls. Equation (18) teaches us the applicable limitation of the present analysis, that is, the Reynolds number $R e$ must be smaller than about 4 for $u_{0} \leq 1$ and $\alpha \leq 60^{\circ}$; this bound of $\alpha$ suffices for practical use as in the present paper. It is also the key factor from which we can derive some reasonable formulae, such as extended Bernoulli equation including friction loss in an exact form and friction coefficient for the Jeffery-Hamel flow. We can understand that from Eq. (18) that $u_{0}$ is constant along the symmetrical axis of $\phi=0$, since $\operatorname{Re}=|Q| /(v \rho)=$ constant and $\alpha=$ constant for a given flow condition. Therefore, the maximum velocity $v_{\max }$ along the symmetrical axis of the channel varies inversely proportional to $r$.

Integrating Eq. (4) together with Eqs. (5), (9), (17), and (18) leads to the extended Bernoulli equation as follows:

$$
\frac{1}{2} \rho v^{2}+p+H=p_{\infty}
$$

where $p_{\infty}$ is the pressure of the quiescent fluid at infinity, i.e., $r \rightarrow \infty$ and the third term of the left-hand side of Eq. (19), $H$, is the friction loss term:

$$
H(r, \phi)=\frac{6 \rho v^{2}}{r^{2}}\left(\frac{2-\alpha^{2}}{2 \alpha^{3}} R e-2 u-3 u^{2}\right)
$$

Equation (19) is equivalent to Eq. (10), but it is expressed in the form of Bernoulli equation containing the friction loss for convenience of discussion after Subsection 2.4. It is the extended Bernoulli equation for the Jeffery-Hamel flow. It reduces to the well-known Bernoulli equation for an ideal fluid in the case of $v=0$, i.e., $H=0$ as it should do. We can understand from Eq. (20) that the friction loss term $H(r, \phi)$ is dependent on not only the Reynolds number $R e$, but also the angle $\alpha$, the distance $r$ from the origin, and a streamline prescribed by the angle $\phi$. Its $r$-dependency is due to the fact that the velocity increases in inverse proportion to the distance. These characteristics are remarkably different from the two-dimensional parallel flow. In the following, we will look into two cases for the friction loss along both the symmetrical channel axis at $\phi=0$ and the channel walls at $\phi= \pm \alpha / 2$.

Rewriting Eq. (20) with the use of Eq. (5), we have

$$
H(r, \phi)=\frac{3 \rho v^{2}\left(2-\alpha^{2}\right)}{\alpha^{3} r^{2}} R e-\frac{2 \rho v}{r} v-\frac{1}{2} \rho v^{2}
$$

For clarity of the meaning of Eq. (21), let us denote the friction loss from infinity to the distance $r$ along both the symmetrical axis and the walls by new symbols $H_{c}$ and $H_{w}$, respectively. Along the symmetrical axis where the velocity is $v(\phi=0)=-6 v u_{0} / r<0, H_{c}$ becomes with the use of $u_{0}=\operatorname{Re} /(4 \alpha)$

$$
H_{c}=H(r, 0)=\frac{6 \rho v^{2}}{\alpha^{3} r^{2}} R e-\frac{1}{2} \rho v^{2} .
$$

On the other hand, along the channel walls where $v(\phi= \pm \alpha / 2)=0, H_{w}$ becomes

$$
H_{w}=H(r, \pm \alpha / 2)=\frac{6 \rho v^{2}}{\alpha^{3} r^{2}} R e-\frac{3 \rho v^{2}}{\alpha r^{2}} R e
$$

Comparison between Eqs. (22) and (23) shows that the friction loss along the axis is larger than the ones along the walls by $3 \rho v^{2} \operatorname{Re} /\left(\alpha r^{2}\right)-\rho v^{2} / 2$ at a distance $r$ from the origin $O$. This difference in the friction loss is due to the fact that the flow velocity is not zero along the axis $\left(v(\phi=0)=-6 v u_{0} / r \neq 0\right)$, while it is zero along the walls $(v(\phi= \pm \alpha / 2)=0)$.

\subsection{Velocity distribution}

The function $u(\phi)$ on a cross section at a position $r$ in the channel can be obtained from Eq. (9). Differentiating Eq. (9) with respect to $\phi$, we have the nonlinear ordinary differential equation as follows:

$$
u^{\prime \prime \prime}+12 u u^{\prime}+4 u^{\prime}=0 \text {. }
$$


The boundary conditions and the condition at the axis to be imposed are

$$
u(\phi=0)=-u_{0}, u\left(\phi=\frac{\alpha}{2}\right)=0,\left.\frac{\mathrm{d} u}{\mathrm{~d} \phi}\right|_{\phi=0}=0
$$

where $u_{0}(>0)$ is given by Eq. (18), i.e., $u_{0}=\operatorname{Re}(4 \alpha)$. Equation (24) can be solved under the above conditions by the optimal homotopy asymptotic method (Esmaeilpour and Ganji, 2010). Its first-order approximate solution $u^{(1)}$ is given by

$$
u^{(1)}=-u_{0}\left[1-4\left(\frac{\phi}{\alpha}\right)^{2}\right]+C u_{0}\left[\frac{1}{15}\left(12 u_{0}-5\right) \phi^{2}-\frac{4}{3}\left(3 u_{0}-1\right) \frac{\phi^{4}}{\alpha^{2}}+\frac{16}{5} u_{0} \frac{\phi^{6}}{\alpha^{4}}\right]
$$

where the leading term of the right-hand side is the zeroth-order approximate solution of Eq. (24), the second term is the first order correction term, and the coefficient $C$ is a constant and can be determined according to Esmaeilpour and Ganji (2010) as follows. The residual $R(\phi, C)$ of the first-order approximate solution $u^{(1)}$ to the exact one of Eq. (24) is expressed as

$$
R(\phi, C)=\frac{d^{3} u^{(1)}}{d \phi^{3}}+12 u^{(1)} \frac{d u^{(1)}}{d \phi}+4 \frac{d u^{(1)}}{d \phi} .
$$

The constant $C$ is determined so that the following functional $J(C)$ can be a minimum value:

$$
J(C)=\int_{-\alpha / 2}^{\alpha / 2} R^{2}(\phi, C) d \phi
$$

The constant $C$ must then satisfy the following condition:

$$
\frac{d J(C)}{d C}=0
$$

The concrete determination of $C$ will be done for special examples in Section 3. Finally, we obtain the first-order approximate solution of the velocity at the position $(r, \phi)$ from Eqs. (5), (18), and (26) as follows:

$$
v^{(1)}(r, \phi)=v^{(0)}(r, \phi)+v^{\prime}(r, \phi),
$$

where

$$
\begin{aligned}
& v^{(0)}(r, \phi)=-\frac{3 v \operatorname{Re}}{2 \alpha r}\left[1-4\left(\frac{\phi}{\alpha}\right)^{2}\right] \\
& v^{\prime}(r, \phi)=\frac{3 v \operatorname{Re} C}{2 \alpha r}\left[\frac{1}{5}\left(\operatorname{Re}-\frac{5}{3} \alpha\right) \frac{\phi^{2}}{\alpha}-\left(\operatorname{Re}-\frac{4}{3} \alpha\right) \frac{\phi^{4}}{\alpha^{3}}+\frac{4}{5} \operatorname{Re} \frac{\phi^{6}}{\alpha^{5}}\right] .
\end{aligned}
$$

The leading term of the right-hand side of Eq. (30), i.e., Eq. (31), denotes the parabolic profile of velocity at the distance $r$ from the origin $O$, which corresponds to that of the two-dimensional parallel flow; the variable of the parabolic form is $\phi$ in this case, while it is the variable normal to the walls in parallel case. Equation (32) means the deviation from the parabolic form. It is found that the magnitude of the velocity in the Jeffery-Hamel flow varies in inverse proportion to the distance $r$ from the origin $O$.

\subsection{Friction loss in finite region}

For applications of above results to actual situations, we will here apply the extended Bernoulli equation (19) to a finite region of channel. The extended Bernoulli equation along a streamline for a finite length of channel, $L$, from $r=r_{1}$ to $r=r_{2}$ as shown in Fig. 2 is from Eq. (19) 


$$
\Delta p=\Delta H+\Delta K
$$

where $\Delta p=p_{1}-p_{2}(>0)$ is the change in static pressure between the positions at $r=r_{1}$ and $r=r_{2}, \Delta H=H_{2}-H_{1}$ ( $>0)$ is the friction loss, and $\Delta K=\rho v_{2}^{2} / 2-\rho v_{1}^{2} / 2(>0)$ is the increase in kinetic energy. Then, the friction loss along a streamline ( $\phi=$ constant, and then $u=$ constant) is from Eq. (21)

$$
\Delta H=H\left(r_{2}, \phi\right)-H\left(r_{1}, \phi\right)=3 \rho v^{2}\left[\frac{2-\alpha^{2}}{\alpha^{3}} R e-2 u(2+3 u)\right]\left(\frac{1}{r_{2}^{2}}-\frac{1}{r_{1}^{2}}\right) .
$$

Therefore, the friction loss $\Delta H_{c}$ along the axis $(\phi=0)$ is given by

$$
\Delta H_{c}=H\left(r_{2}, 0\right)-H\left(r_{1}, 0\right)=\frac{3 \rho v^{2} R e}{\alpha^{3}}\left(2-\frac{3}{8} \alpha R e\right)\left(\frac{1}{r_{2}^{2}}-\frac{1}{r_{1}^{2}}\right),
$$

on the other hand, the friction loss $\Delta H_{w}$ along the walls $(\phi= \pm \alpha / 2)$ is

$$
\Delta H_{w}=H\left(r_{2}, \pm \alpha / 2\right)-H\left(r_{1}, \pm \alpha / 2\right)=\frac{3 \rho v^{2} R e}{\alpha^{3}}\left(2-\alpha^{2}\right)\left(\frac{1}{r_{2}^{2}}-\frac{1}{r_{1}^{2}}\right) \text {. }
$$

For the purpose of the comparison between the above friction losses and that of the two-dimensional parallel flow not depending on $\phi$, it is convenient for us to express the former ones by cross-sectional quantities. Here, we will define the cross-sectional average of arbitrary function $F(r, \phi)$ as

$$
\bar{F}(r)=\frac{1}{\alpha} \int_{-\alpha / 2}^{\alpha / 2} F(r, \phi) d \phi
$$

Denoting the average friction loss (the friction loss averaged on the cross-section at $r$ ) by $\Delta \bar{H}$, we obtain from Eq. (21) or (34)

$$
\Delta \bar{H}=\bar{H}\left(r_{2}\right)-\bar{H}\left(r_{1}\right)=\frac{3 \rho v^{2} \operatorname{Re}}{\alpha^{3}}\left(2-\frac{1}{3} \alpha^{2}-\frac{1}{5} \alpha \operatorname{Re}\right)\left(\frac{1}{r_{2}^{2}}-\frac{1}{r_{1}^{2}}\right) .
$$

Similarly, the average velocities are

$$
\begin{aligned}
& \bar{v}(r)=-\frac{v R e}{\alpha r}=\frac{2}{3} v_{\max }, \\
& \overline{v^{2}}(r)=-\frac{6 v^{2} R e^{2}}{5 \alpha^{2} r^{2}}=\frac{8}{15} v_{\max }^{2},
\end{aligned}
$$

where the parabolic profile, i.e., Eq. (31) has been used and $v_{\max }$ is flow velocity on the symmetrical axis.

Expressing Eqs. (35), (36), and (38) by the ratios to the friction loss $\Delta H^{\text {para }}$ of the two-dimensional parallel flow is convenient for comparison among them, where $\Delta H^{\text {para }}$ is given by

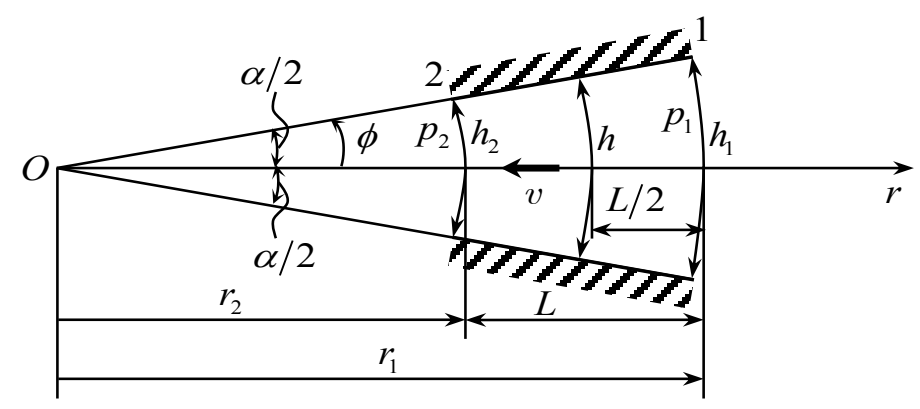

Fig. 2 Symbols in the finite region of channel. The walls are hatched by oblique lines and their axial length is denoted by $L$. 


$$
\Delta H^{\text {para }}=\frac{12 v L|Q|}{h^{3}}
$$

in which $|Q|$ is the mass flow rate, $L$ is the channel length, and $h$ is the parallel channel width (Levich, 1962; Schlichting, 1968). Here, we put the width $h$ to be the arithmetical average of widths at the inlet and the exit of the channel, i.e.,

$$
h=\frac{r_{1}+r_{2}}{2} \alpha
$$

Then, the ratios of Eqs. (35), (36), and (38) to $\Delta H^{\text {para }}$ are represented as

$$
\begin{aligned}
& \frac{\Delta H_{c}}{\Delta H^{\text {para }}}=\left(1-\frac{3}{16} \alpha R e\right) \times \frac{1}{16}\left(\frac{r_{2}}{r_{1}}+\frac{r_{1}}{r_{2}}+2\right)^{2}, \\
& \frac{\Delta H_{w}}{\Delta H^{\text {para }}}=\left(1-\frac{1}{2} \alpha^{2}\right) \times \frac{1}{16}\left(\frac{r_{2}}{r_{1}}+\frac{r_{1}}{r_{2}}+2\right)^{2}, \\
& \frac{\Delta \bar{H}}{\Delta H^{\text {para }}}=\left(1-\frac{1}{6} \alpha^{2}-\frac{1}{10} \alpha R e\right) \times \frac{1}{16}\left(\frac{r_{2}}{r_{1}}+\frac{r_{1}}{r_{2}}+2\right)^{2} .
\end{aligned}
$$

Equations (43) - (45) all become unity when $\alpha$ tends to zero as they should do. The above results certainly demonstrate that the present theory can recover the well-known friction loss in the case of $\alpha=0$, i.e., $\Delta H^{\text {para }}$ given by Eq. (41). In the next section, we will also derive the well-known friction coefficient for the two-dimensional parallel flow, i.e., 24/Re, from Eq. (45). Equations (43) - (45) show that the friction losses $\Delta H_{c}, \Delta H_{w}$, and $\Delta \bar{H}$ are dependent on not only the Reynolds number $R e$, but also the angle $\alpha$ and the positions $r=r_{1}$ and $r=r_{2}$; this is the point of the present results remarkably different from the parallel flow.

Finally, for reference, the ratio of the pressure change $\Delta p_{\text {ideal }}$, which equals to the kinetic energy increase $\Delta K$ along the axis for ideal fluid, to $\Delta H^{\text {para }}$ can be given from the Bernoulli equation for the ideal fluid as

$$
\frac{\Delta p_{\text {ideal }}}{\Delta H^{\text {para }}}=\frac{3}{16} \alpha \operatorname{Re} \times \frac{1}{16}\left(\frac{r_{2}}{r_{1}}+\frac{r_{1}}{r_{2}}+2\right)^{2}
$$

although there is no friction loss in ideal fluid. Quantitative evaluation of Eqs. (43) - (46) will be illustrated in the next section. It should be once again noted that all results above hold for a divergent flow if the sign of all velocities except $u_{0}$ is inverted.

\section{Quantitative evaluation of velocity distribution, friction loss, and friction coefficient}

We will here discuss concretely the velocity distribution, the friction losses, and the friction coefficient for the configuration of the finite channel region as $\alpha=32^{\circ}, r_{1}=0.32 \mathrm{~mm}$, and $L=r_{1}-r_{2}=0.16 \mathrm{~mm}\left(r_{2}=0.16 \mathrm{~mm}\right)$; then $h_{1}=r_{1} \alpha=0.179 \mathrm{~mm}$. These dimensions were read from the image reconstructed by CAD of the visualized lymphatic vessel of a mouse. Fluid is water, and its density and kinematic viscosity are respectively $\rho=1000 \mathrm{~kg} / \mathrm{m}^{3}$ and $v=1.0 \times 10^{-6} \mathrm{~m}^{2} / \mathrm{s}$. The dimensionless velocity $u_{0}$ (=constant) is taken to be 0.5 , and then, the mass flow rate $|Q|$ is estimated by $4 \rho v \alpha u_{0}$ from the Reynolds number $(R e=|Q| /(\rho v))$ and Eq. (18); for example, $|Q|=1.12 \times 10^{-3} \mathrm{~kg} / \mathrm{s}$ for $\alpha=32^{\circ}$ and $|Q|=2.09 \times 10^{-3} \mathrm{~kg} / \mathrm{s}$ for $\alpha=60^{\circ}$. The open ratio of the convergent channel, $\beta$, is defined as the ratio of cross section area at the exit, i.e., at $r=r_{2}$ to the one at the angle $\alpha=32^{\circ}$ where $\beta=1$. We look into details of the flow in the range from $\alpha=32^{\circ}(\beta=1)$ to $\alpha=60^{\circ}(\beta=0.125)$. Note that $r_{1}$ and $r_{2}$ change according to the shift of the origin when $\alpha$ varies keeping $L$ and $h_{1}$ fixed.

Figure 3 shows both (a) the zeroth- and the first-order velocity distributions and (b) the deviation from the zeroth-order approximation of the Jeffery-Hamel flow, which are respectively represented by Eqs. (31), (30), and (32) at $r=r_{2}$ for $u_{0}=0.5$, and the convergent angles $\alpha=32^{\circ}, 40^{\circ}, 48^{\circ}$, and $56^{\circ}$ which respectively correspond to $\beta=1,0.75,0.5$, and 0.25 . Both the channel inlet width $h_{1}=0.179 \mathrm{~mm}$ and the axial length of the channel 
$L=0.16 \mathrm{~mm}$ at $\alpha=32^{\circ}, r_{1}=0.32 \mathrm{~mm}$, and $r_{2}=0.16 \mathrm{~mm}$ are fixed. The velocity distributions of the first-order approximate solution, as shows in Fig. 3(a), are all just parabolic shapes. The difference between the zeroth- and the first-order velocity distributions is so small that it is not appreciable. The constant $C$ in Eq. (26) under the present conditions is almost equal to -1 : its numerical evaluation is from -0.99983 to -0.99945 depending on values of $\alpha$ from $32^{\circ}$ to $56^{\circ}$.

Figure 4 shows the friction loss ratios $\Delta H_{c} / \Delta H^{\text {para }}$ (red), $\Delta H_{w} / \Delta H^{\text {para }}$ (blue), $\Delta \bar{H} / \Delta H^{\text {para }}$ (purple), and the pressure change ratio $\Delta p_{\text {ideal }} / \Delta H^{\text {para }}$ (black) versus the convergent angle $\alpha$ and the open ratio $\beta$ under the same conditions as in Fig. 3: $\Delta H_{c} / \Delta H^{\text {para }}$ is given by Eq. (43), $\Delta H_{w} / \Delta H^{\text {para }}$ by Eq. (44), $\Delta \bar{H} / \Delta H^{\text {para }}$ by Eq. (45), and $\Delta p_{\text {ideal }} / \Delta H^{\text {para }}$ by Eq. (46). The lower abscissa of the figure denotes the convergent angle $\alpha$, while the upper one indicates the open ratio $\beta$. It is found that $\Delta H_{c} / \Delta H^{\text {para }}, \Delta H_{w} / \Delta H^{\text {para }}$, and $\Delta \bar{H} / \Delta H^{\text {para }}$ are all larger than unity, and this means that all three friction losses are larger than that of the two-dimensional parallel flow. In particular, they become extremely large as $\alpha$ approaches $\alpha=60^{\circ}(\beta=0.125)$. When $\alpha \rightarrow 0$, all of $\Delta H_{c} / \Delta H^{\text {para }}, \Delta H_{w} / \Delta H^{\text {para }}$, and $\Delta \bar{H} / \Delta H^{\text {para }}$ reduce to unity as it should do. The ratio $\Delta p_{\text {ideal }} / \Delta H^{\text {para }}$ is smaller than all of $\Delta H_{c} / \Delta H^{\text {para }}$, $\Delta H_{w} / \Delta H^{\text {para }}$, and $\Delta \bar{H} / \Delta H^{\text {para }}$. The reason why $\Delta H_{c} / \Delta H^{\text {para }}, \Delta H_{w} / \Delta H^{\text {para }}$, and $\Delta \bar{H} / \Delta H^{\text {para }}$ are larger than unity is due to that the flow velocity is accelerated inversely proportional to the distance $r$ as it approaches to the sink at the origin $O$.

Finally, the friction coefficient of the Jeffery-Hamel flow will be derived from Eq. (38). We express the average friction loss $\Delta \bar{H}$ as $\Delta \bar{H}=\lambda(L / h)\left(\rho \bar{v}^{2} / 2\right)$ and the friction coefficient $\lambda$ as

$$
\lambda=\frac{\Pi}{R e} .
$$

Furthermore, defining $\Delta H^{\text {para }}$ as $\Delta H^{\text {para }}=(24 / R e)(L / h)\left(\rho \bar{v}^{2} / 2\right)$, we have from Eq. (45)

$$
\Pi=24\left(1-\frac{1}{6} \alpha^{2}-\frac{1}{10} \alpha \operatorname{Re}\right) \times \frac{1}{16}\left(\frac{r_{2}}{r_{1}}+\frac{r_{1}}{r_{2}}+2\right)^{2} .
$$

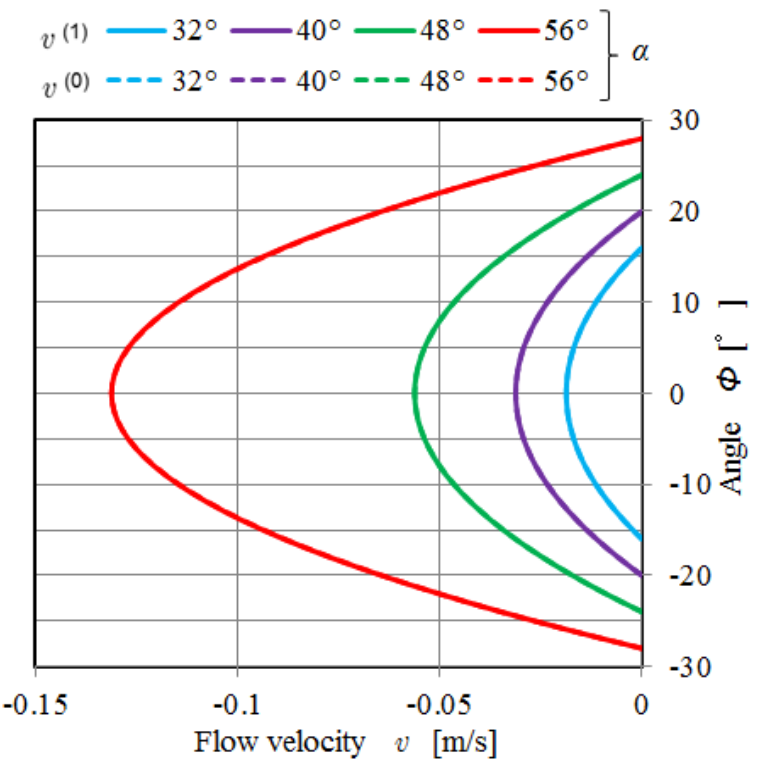

(a) The zeroth- and the first-order approximate solutions

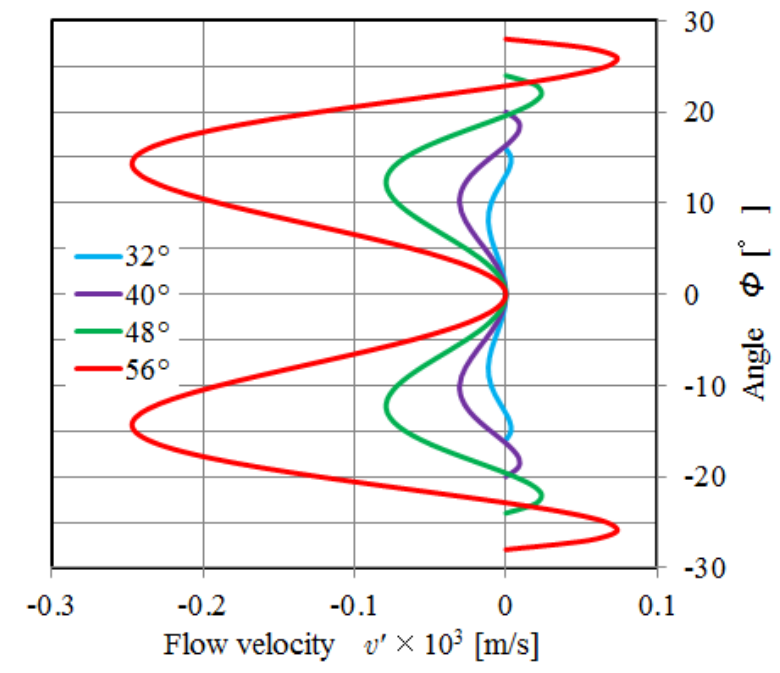

(b) Deviation from the zeroth-order approximate solution

Fig. 3 (a)The zeroth- and the first-order approximate solutions (Eqs. (31) and (30), respectively) and (b)deviation (Eq. (32)) from the zeroth-order approximate solution at the exit of the channel for $u_{0}=0.5$, and the convergent angles $\alpha=32^{\circ}, 40^{\circ}, 48^{\circ}$, and $56^{\circ}$ corresponding to $\beta=1,0.75,0.5$, and 0.25 , respectively. The first-order approximate solution forms parabolic profiles in the same way as the two-dimensional parallel flow. The deviation from the zeroth-order approximate solution is described by the sixth-degree polynomial, though it is too small to be discernible in (a). 


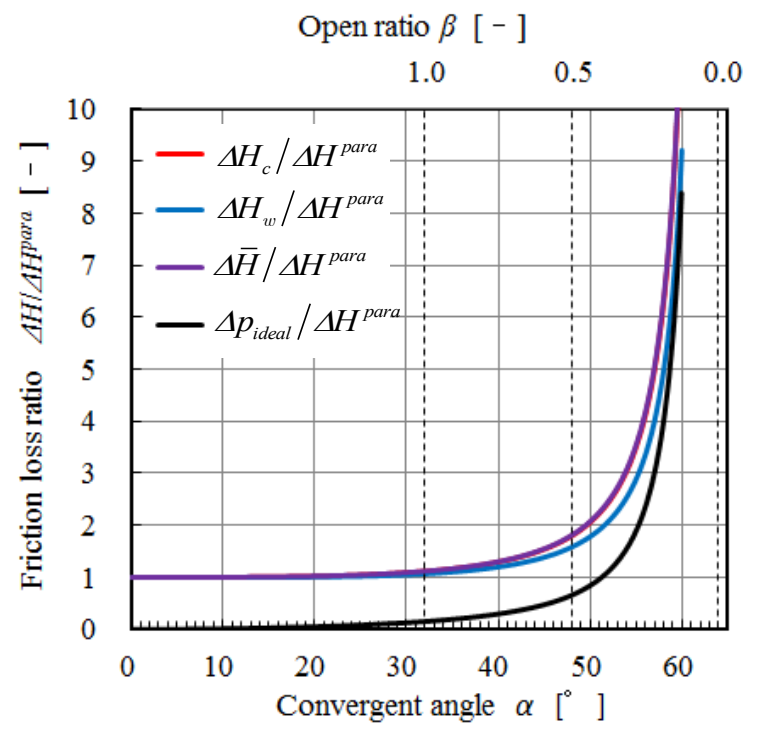

Fig. 4 The friction loss ratios $\Delta H_{c} / \Delta H^{\text {para }}$ (red, Eq. (43)), $\Delta H_{w} / \Delta H^{\text {para }}$ (blue, Eq. (44)), $\Delta \bar{H} / \Delta H^{\text {para }}$ (purple, Eq. (45)), and the pressure change ratio $\Delta p_{\text {ideal }} / \Delta H^{\text {para }}$ (black, Eq. (46)) versus the convergent angle $\alpha$ and the open ratio $\beta$.

The function $\Pi$ given by Eq. (48) is dependent on the geometrical configuration of $\alpha, r_{1}$, and $r_{2}$. Figure 5 shows the profile of the function $\Pi$ for the convergent angle $\alpha$ under the same conditions as in Figs. 3 and 4 . The dependency of $\Pi$ on $\alpha$ is similar to those of the friction losses shown in Fig. $4 . \Pi$ is just 24 at $\alpha=0$ as it should be and increases exponentially as the angle $\alpha$ does.

Figure 6 shows effects of the channel length on the friction loss ratios $\Delta H_{c} / \Delta H^{\text {para }}, \Delta H_{w} / \Delta H^{\text {para }}, \Delta \bar{H} / \Delta H^{\text {para }}$ and the pressure change $\Delta p_{\text {ideal }} / \Delta H^{\text {para }}$ at $\alpha=32^{\circ}, r_{2}=0.16 \mathrm{~mm}$, and $u_{0}=0.5$. For example, when the channel length $L$ is 2 times of $r_{2}$, both the friction losses along the axis and along the walls are larger than that of the two-dimensional parallel flow by about 1.57 and 1.50 times, respectively. Generally, the friction losses of the Jeffery-Hamel flow are larger than that of the two-dimensional parallel flow at $L \geq r_{2}$, and their discrepancy becomes larger according to the increase in the channel length. The pressure change in the ideal fluid flow is smaller than that of the two-dimensional parallel flow, i.e., $\Delta p_{\text {ideal }} / \Delta H^{\text {para }}<1$, and is less susceptible to the channel length.

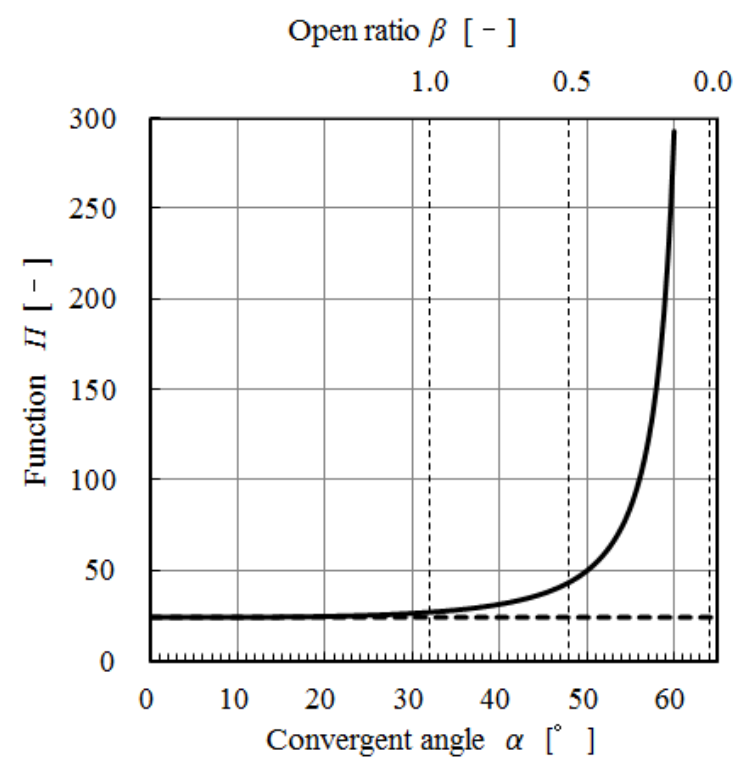

Fig. 5 The variation of $\Pi$, i.e., Eq. (48) for the convergent angle $\alpha$ under the same conditions as in Figs. 3 and 4. $\Pi$ reasonably approaches to 24 for the two-dimensional parallel flow in the limit of $\alpha \rightarrow 0$. 


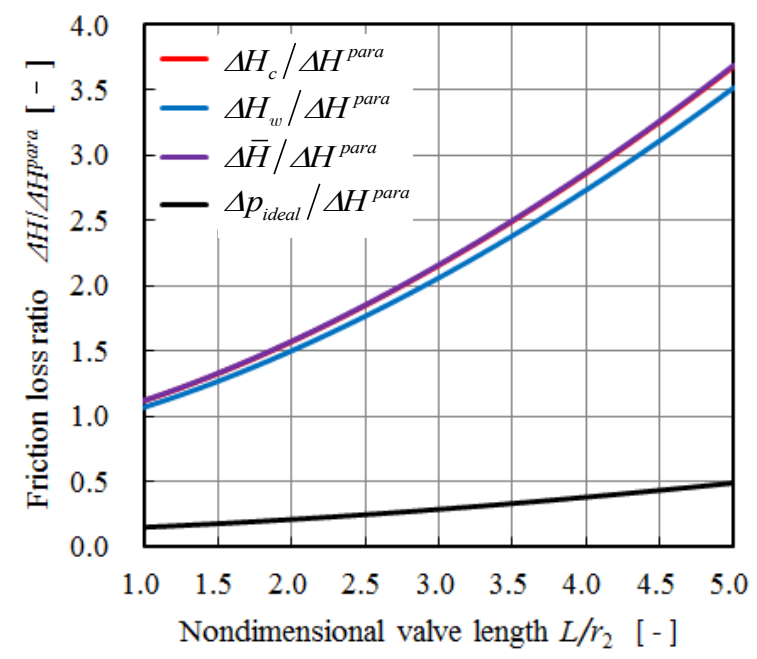

Fig. 6 The friction loss ratios along the symmetrical axis (red, Eq. (43)) and along the walls (blue, Eq. (44)), cross-sectional average friction loss (purple, Eq. (45)), and the pressure change for ideal fluid (black, Eq. (46)) versus the dimensionless channel length $L / r_{2}$. All friction losses are larger than that of the two-dimensional parallel flow at $L \geq r_{2}$ and their discrepancy becomes larger according to the increase in $L / r_{2}$.

\section{Conclusions}

The steady two-dimensional microscopic flow of small Reynolds number between two semi-infinite convergent planes has been analyzed in a theoretical way. The basic relation among the convergent or divergent angle $\alpha$, the dimensionless velocity $u_{0}$, and the Reynolds number $R e$ has been obtained in the form of $R e=4 \alpha u_{0}$ which is another expression different from the equation given in Landau and Lifshitz's textbook for large Reynolds number. This relation also provides the applicable limitation of the present theory, that is, the Reynolds number $R e$ must be smaller than about 4 for $u_{0} \leq 1$ and $\alpha \leq 60^{\circ}$. The extended Bernoulli equation, the friction loss, and the friction coefficient have been obtained in exact forms and the zeroth- and first-order approximate solutions of velocity distribution have also been derived by the optimal homotopy asymptotic method. It has been clarified that the friction losses and the friction coefficient in the finite region of the channel have been expressed by the explicit functions of the convergent or divergent angle and they greatly increase according to the increases in the angle. The friction coefficient $\lambda$ in the finite region has been defined suitably and its simple formula has been derived. The coefficient is reasonably identical with 24/Re for the two-dimensional parallel flow as the special case of $\alpha \rightarrow 0$; this means that the friction coefficient ensured by the relation $R e=4 \alpha u_{0}$ is reasonable. Although the present theory is limited for microscopic and small Reynolds number flows, some formulae obtained can be used for estimating the friction losses and the friction coefficient for a type of Jeffery-Hamel flow. The authors are now trying to extend the present theory to some more realistic cases such as a flow between permeable planes and an axi-symmetric flow in analytical ways. A CFD analysis is also being performed for the axi-symmetric case.

\section{Acknowledgment}

This study was supported in part by JSPS KAKENHI Grant Numbers, 26242051 (TK) and 24650286 (TK).

\section{References}

Banks, W. H. H., Drazin, P. G. and Zaturska, M. B., On perturbations of Jeffery-Hamel flow, Journal of Fluid Mechanics, Vol. 186 (1988), pp. 559-581.

Esmaeilpour, M. and Ganji, D. D., Solution of the Jeffery-Hamel flow problem by optimal homotopy asymptotic method, Computers and Mathematics with Applications, Vol. 59, No. 11 (2010), pp. 3405-3411.

Goldshtik, M., Hussain, F. and Shtern, V., Symmetry breaking in vortex-source and Jeffery-Hamel flows, Journal of 
Fluid Mechanics, Vol. 232 (1991), pp. 521-566.

Haines, P. E., Hewitt, R. E. and Hazel, A. L., The Jeffery-Hamel similarity solution and its relation to flow in a diverging channel, Journal of Fluid Mechanics, Vol. 687 (2011), pp. 404-430.

Hamel, G., Spiralförmige Bewegungen zäher Flüssigkeiten, Jahresbericht der Deutschen Mathematiker-Vereinigung, Vol. 25 (1916), pp. 34-60.

Jeffery, G. B., The two-dimensional steady motion of a viscous fluid, Philosophical Magazine Series 6, Vol. 29, No. 172 (1915), pp. 455-465.

Kato, S., Shirai, Y., Kanzaki, H., Sakamoto, M., Mori, S. and Kodama, T., Delivery of molecules to the lymph node via lymphatic vessels using ultrasound and nano/microbubbles, Ultrasound in Medicine and Biology, Vol. 41, No. 5 (2015), pp. 1411-1421.

Khan, U., Ahmed, N., Zaidi, Z. A., Jan, S. U. and Mohyud-Din, S. T., On Jeffery-Hamel flows, International Journal of Modern Mathematical Sciences, Vol. 7, No. 3 (2013), pp. 236-247.

Kodama, T., Hatakeyama, Y., Kato, S. and Mori, S., Visualization of fluid drainage pathways in lymphatic vessels and lymph nodes using a mouse model to test a lymphatic drug delivery system, Biomedical Optics Express, Vol. 6, No. 1 (2015), pp. 124-134.

Kodama, T., Matsuki, D., Tada, A., Takeda, K. and Mori, S., New concept for the prevention and treatment of metastatic lymph nodes using chemotherapy administered via the lymphatic network, Scientific Reports, Vol. 6 (2016), 32506.

Landau, L. D. and Lifshitz, E. M., Fluid Mechanics, 2nd ed. (1987), Pergamon Press.

Levich, V. G., Physicochemical Hydrodynamics (Translated by Scripta Technica, Inc.), 2nd ed. (1962), Prentice-Hall, Inc.

Millsaps, K. and Pohlhausen, K., Thermal distributions in Jeffery-Hamel flows between nonparallel plane walls, Journal of the Aeronautical Sciences, Vol. 20, No. 3 (1953), pp. 187-196.

Okuno, T., Kato, S., Hatakeyama, Y., Okajima, J., Maruyama, S., Sakamoto, M., Mori, S. and Kodama, T., Photothermal therapy of tumors in lymph nodes using gold nanorods and near-infrared laser light, Journal of Controlled Release, Vol. 172, No. 3 (2013), pp. 879-884.

Sabersky, R. H., Acosta, A. J. and Hauptmann, E. G., Fluid Flow - A First Course in Fluid Mechanics, 2nd ed. (1971), Macmillan Publishing Co., Inc.

Sato, T., Mori, S., Arai, Y. and Kodama, T., The combination of intralymphatic chemotherapy with ultrasound and nano-/microbubbles is efficient in the treatment of experimental tumors in mouse lymph nodes, Ultrasound in Medicine and Biology, Vol. 40, No. 6 (2014), pp. 1237-1249.

Schlichting, H., Boundary-Layer Theory (Translated by Kestin, J.), 6th ed. (1968), McGraw-Hill, Inc.

Shao, L., Mori, S., Yagishita, Y., Okuno, T., Hatakeyama, Y., Sato, T. and Kodama, T., Lymphatic mapping of mice with systemic lymphoproliferative disorder: Usefulness as an inter-lymph node metastasis model of cancer, Journal of Immunological Methods, Vol. 389, No. 1-2 (2013), pp. 69-78.

Stow, S. R., Duck, P. W. and Hewitt, R. E., Three-dimensional extensions to Jeffery-Hamel flow, Fluid Dynamics Research, Vol. 29, No. 1 (2001), pp. 25-46. 